\title{
Does COVID-19 Disease Affect Dental Implant Success
}

\section{Rate?}

\author{
Orkhan Ismayilov ${ }^{1 *}$, Ali Ekemen ${ }^{1}$ and Hakan Alpay ${ }^{2}$ \\ ${ }^{1}$ Faculty of Dentistry, Assistant professor, Department of Oral and Maxillofacial Surgery, Ankara Medipol University, \\ Turkey \\ ${ }^{2}$ Faculty of Dentistry, Assistant professor, Department of Oral and Maxillofacial Surgery, Ankara Medipol University, \\ Turkey
}

\section{Introduction}

COVID-19 pandemics, spreading rapidly all over the world continues to affect the world even though vaccines are being applied [1]. The authors of this study recently observed that the dental implant success rates decrease in patients with COVID-19 disease after dental implant surgery. In a group of 14 patients, 85 dental implants were placed, and 4 of the implants were failed. It was observed that all the patients with failed implants had a positive COVID-19 test 4-6 weeks after the surgery. Six of the 14 patients were affected by COVID-19 and 4 out of 42 implants applied to these patients were failed. All implants were placed by the same surgeon using the same brand of an implant with appropriate primary stability. Only one of these patients had pain during the recovery period, but all other 5 patients with implant failure have no symptoms. Radiological examination reveals neck resorption in only one of the 4 failed implants. This situation brought to the mind of the authors that COVID-19 disease could have negative effects on osteointegration and the success rate of dental implant surgery. After a review of the recent literature, clinically similar results were explained, stating COVID-19 disease may negatively affect bone osseointegration [2].

\section{Mechanisms}

It has been reported in the literature that COVID-19 disease may affect the skeletal system by several mechanisms [3]. Angiotensin-converting enzyme 2 (ACE2) can induce bone resorption. ACE2 is a receptor for the spike glycoprotein of COVID-19 based on the high degree of homology of the receptor-binding domain between COVID-19 and SARSCoV [4]. Also, ACE2 is an enzyme of an important reninangiotensin system [5] that catalyzes the separation of angiotensin II to Ang-(1-7) and then targets MasR. Recent studies have demonstrated that ACE2 and MasR are expressed by osteoblasts and osteoclasts, while activation of the ACE2 / Ang-(1-7) / MasR pathway can reduce the bone resorption environment by inhibiting the expression of the nuclear factor receptor activator (RANKL). Therefore, when COVID-19 targets ACE2, it weakens the ACE2 / Ang- (1-7) / MasR cascade by reducing ACE2 expression, and by improper bone homeostasis, both osteoblast genesis and osteoblast genesis processes are accelerated [6]. According to another mechanism, inflammatory factors and cytokine storm may be a vital cause of bone loss. High amounts of inflammatory cytokines such as IL-1, IL-6 TNF- $\alpha$, G-CSF, IP-10, MCP-1, MIP$1 \alpha$ have been detected in patients infected with COVID-19, especially in need of intensive care [7]. These factors may play a role in many steps from osteoclast formation to other different aspects of the osteoclastogenic pathway to increase bone resorption capacity through the RANK / RANKL / OPG signaling. Therefore, if the acute high cytokine concentration induced by COVID-19 is not fully controlled and turns into a chronic state; these inflammatory cytokines can trigger osteoclasts, bone loss and inhibit bone formation. At the same time, immunosuppression contributes to bone destruction. During the COVID-19 infection, after the immune system is attacked, the decrease of peripheral blood lymphocytes, especially $T$ and $B$ cells. In such a situation, $T$ cells are activated and transformed into Th17 cells which causing bone destruction [5].

Rovas, et al. stated that new vessels formed during the healing process and rate of blood flow, the hallmarks of microcirculation, decreases in COVID-19 patients. In another study, the authors claimed that patients with COVID-19 developed microvascular inflammation and thrombosis [8].

The negative effects of corticosteroid use on bone mass, osteoclast activity and bone resorption are well known, and corticosteroids are frequently preferred for the treatment of COVID-19 disease [9]. This could have another undesirable effect on bone healing after dental implant surgery.

*Corresponding author: Orkhan Ismayilov, Faculty of Dentistry, Assistant professor, Department of Oral and Maxillofacial Surgery, Ankara Medipol University, Turkey

Accepted: July 22, 2021

Published online: July 24, 2021

Citation: Ismayilov O, Ekemen A, Alpay H (2021) Does COVID-19 Disease Affect Dental Implant Success Rate?. Archives Oral Maxillofac Surg 4(2):126-127 


\section{Conclusion}

With the above-mentioned mechanisms, the authors of this study think that during surgical dental implant procedures, the healing process is affected due to the decrease in osteogenesis resulting with dental implant failure. It could be beneficial to carefully consult patients who have had COVID-19 disease before dental implant surgery and to inform patients about the possibility of a reduced implant success rate. In addition, could be also patients who have not had COVID-19 disease should be told the possibility of a decrease in implant success rate if they have the disease during the recovery period after surgery. Finally, these observations must be evaluated and studied in larger patient groups.

\section{References}

1. Covid-19 vaccines (2021) Organization World Health Organization.

2. Block MS (2021) Coronavirus Disease 2019 May Affect Dental Implant Integration. J Oral and Maxillofacial Surgery 79: 11971198.
3. Zheng K, Zhang W, Xu Y, et al. (2020) COVID-19 and the bone: Underestimated to consider. Eur Rev Med Pharmacol Sci 24: 10316-10318.

4. Wang C, Xu J, Yang L, et al. (2018) Prevalence and risk factors of chronic obstructive pulmonary disease in China (the China Pulmonary Health [CPH] study): a national cross-sectional study. The Lancet. 391: 1706-1717.

5. Okamoto K, Nakashima T, Shinohara M, et al. (2017) Osteoimmunology: The conceptual framework unifying the immune and skeletal systems. Physiol Rev 97: 1295-1349.

6. Queiroz-Junior CM, Santos ACPM, Galvão I, et al. (2019) The angiotensin converting enzyme 2/angiotensin-(1-7)/Mas Receptor axis as a key player in alveolar bone remodeling. Bone 128: 115041.

7. Huang C, Wang Y, Li X, et al. (2020) Clinical features of patients infected with 2019 novel coronavirus in Wuhan, China. The lancet 395: 497-506.

8. Rovas A, Osiaevi I, Buscher K, et al. (2021) Microvascular dysfunction in COVID-19: the MYSTIC study. Angiogenesis 24: 145-157.

9. Russell CD, Millar JE, Baillie JK (2020) Clinical evidence does not support corticosteroid treatment for 2019-nCoV lung injury. The Lancet 395: 473-475. 